Available online on 15.06.2021 at http://jddtonline.info
Copyright (c) 2021 The Author(s): This is an open-access article distributed under the terms of the CC BY-NC
4.0 which permits unrestricted use, distribution, and reproduction in any medium for non-commercial use
provided the original author and source are credited

\title{
(2) \\ Open \\ Unexplained Negative Electromagnetic Radiation and its Reduction effect on Human by Electromagnetic seed Rudraksha
}

\author{
DSubhashish Tripathy*1, (D)Amit Mishra², (D)Arun Kumar Mishra ${ }^{3}$ \\ ${ }^{1}$ Ph.D. Research Scholar IFTM University, Moradabad, India
}

${ }^{2}$ Director Maharana Pratap College of Pharmaceutical Sciences (MPCPS), Kanpur, India

${ }^{3}$ Professor, Faculty of Pharmacy IFTM University, Moradabad, India

\section{Article Info:}

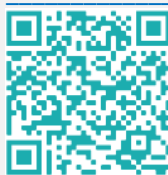

\section{Article History:}

Received 22 April 2021

Reviewed 26 May 2021

Accepted 02 June 2021

Published 15 June 2021

\section{Cite this article as:}

Tripathy S, Mishra A, Mishra AK, Unexplained Negative Electromagnetic Radiation and its Reduction effect on Human by Electromagnetic seed Rudraksha, Journal of Drug Delivery and Therapeutics. 2021; 11(3-S):48-52

DOI: http://dx.doi.org/10.22270/jddt.v11i3-S.4881

\begin{abstract}
Electric and magnetic fields (EMFs) are indefinable regions of energy that are often referred to as Radiation and are linked to the use of electricity as well as various natural and man-made phenomena. Due to continuous exposure to Unexplained and negative EMF, people experienced stress, anxiety, Hypoxia, and even long-term exposure result in Cancer to patients. On the other hand, Rudraksha seeds are globally known as electromagnetic seeds due to their positive electromagnetic healing effect. This research was conducted to find the consequence of the Negative EMF effect on human pulse rate and Oxygen carrying capacity in blood and the use of Rudraksha to study cut off harmful effect of negative EMF by this Electromagnetic property. This topic constitutes a new domain with the largely unstudied potential of Rudraksha seeds shielding effect on Unexplained Negative Electromagnetic Radiation.
\end{abstract}

Keywords: EMF, Rudraksha, electromagnetic seeds, human pulse rate, Oxygen carrying capacity, Negative Electromagnetic Radiation.

\footnotetext{
*Address for Correspondence:

Subhashish Tripathy, Ph.D. Research Scholar IFTM University, Moradabad, India. Email ID: Subhashishpharma@gmail.com
}

ORCID ID : https://orcid.org/0000-0003-3936-3987

\section{INTRODUCTION:}

\subsection{EMF (Electromagnetic Field):}

The motion of electrically charged particles produces electromagnetic waves. Since they radiate from electrically charged particles, these waves are also known as electromagnetic radiation. They can move through space as well as air and other materials ${ }^{1}$. Low-frequency electromagnetic waves are considered electromagnetic fields, whereas high-frequency electromagnetic waves are called electromagnetic radiations. The motion of an electric charge causes an electromagnetic field, which is a property of space ${ }^{2}$. In the surrounding space, a stationary charge can only emit an electric field. As a charge moves, it generates a magnetic field. A shifting magnetic field may also generate an electric field. An electromagnetic field is generated by the mutual interaction of electric and magnetic fields. Electric fields are formed as a result of voltage variations. The greater the resulting field, the higher the voltage. Magnetic fields form as electric current flows show that the greater the current, the stronger the magnetic field ${ }^{3}$. There would be an electric field even though there is no current flowing. As current flows, the strength of the magnetic field varies, but the strength of the electric field remains constant. The electromagnetic spectrum, in addition to natural sources, Human-made sources produce electromagnetic fields as well. EMF is produced when X-rays are used to diagnose a broken limb. Low-frequency electromagnetic fields are correlated with the energy that comes out of a power socket ${ }^{4,} \mathbf{5}$. TV antennas, radio stations, and cell phone base stations all use different types of higher frequency radio waves to transmit EMF.

An electromagnetic field (EMF) is a region of electrical charges that are moving. Some EMFs, especially those involving ionizing radiation, have the potential to be harmful to humanity. EMFs occur on a scale, and the wavelength and frequency of a field determine where it is on the spectrum. There are two types of electromagnetic fields ${ }^{7}$. Ionizing radiation fields have shorter wavelengths and higher frequencies. This form of radiation can remove electrons from atoms, including those found in water and living tissue. Chemical bonds are broken by this EMF. EMFs that aren't ionizing have a longer wavelength and lower frequency ${ }^{8}$. They are incapable of breaking chemical bonds. 


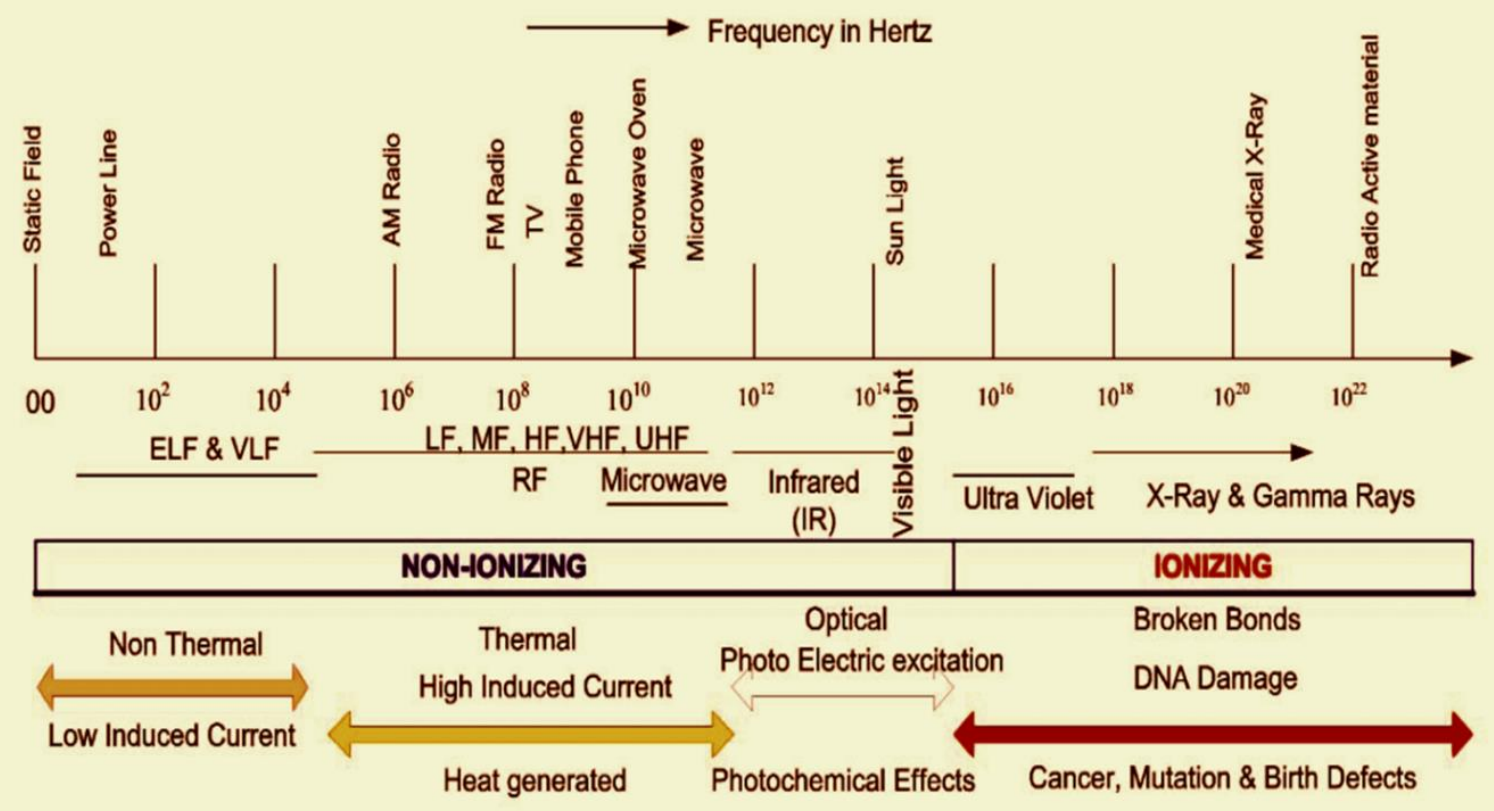

Figure 1: Electromagnetic Spectrum6 ${ }^{6}$

\subsection{Negative EMF and its effect on health:}

Negative EMF In technical terms means a current that opposes the transport in magnetic flux by creating a magnetic field. EMF calculation plays a vital role in the detection of negative Electromagnetic radiation present in the area of our planet. Unexplained and negative EMF does exist in different locality of our earth ${ }^{9}$. Long-term exposure to this EMF causes Stress, Anxiety, depression high pulse rate, and hypoxia-like condition in our body ${ }^{6}$. Long-term exposure to this negative energy even causes Leukaemia in our body and malignant cell develop in our body tissue in a different part of our organ. Negative, unexplained EMFs may harm your body's nervous system and cause cell damage. Symptoms of high EMF exposure may be cancer and irregular growths with other signs and symptoms include sleep disturbances, such as insomnia, and hypoxia10.

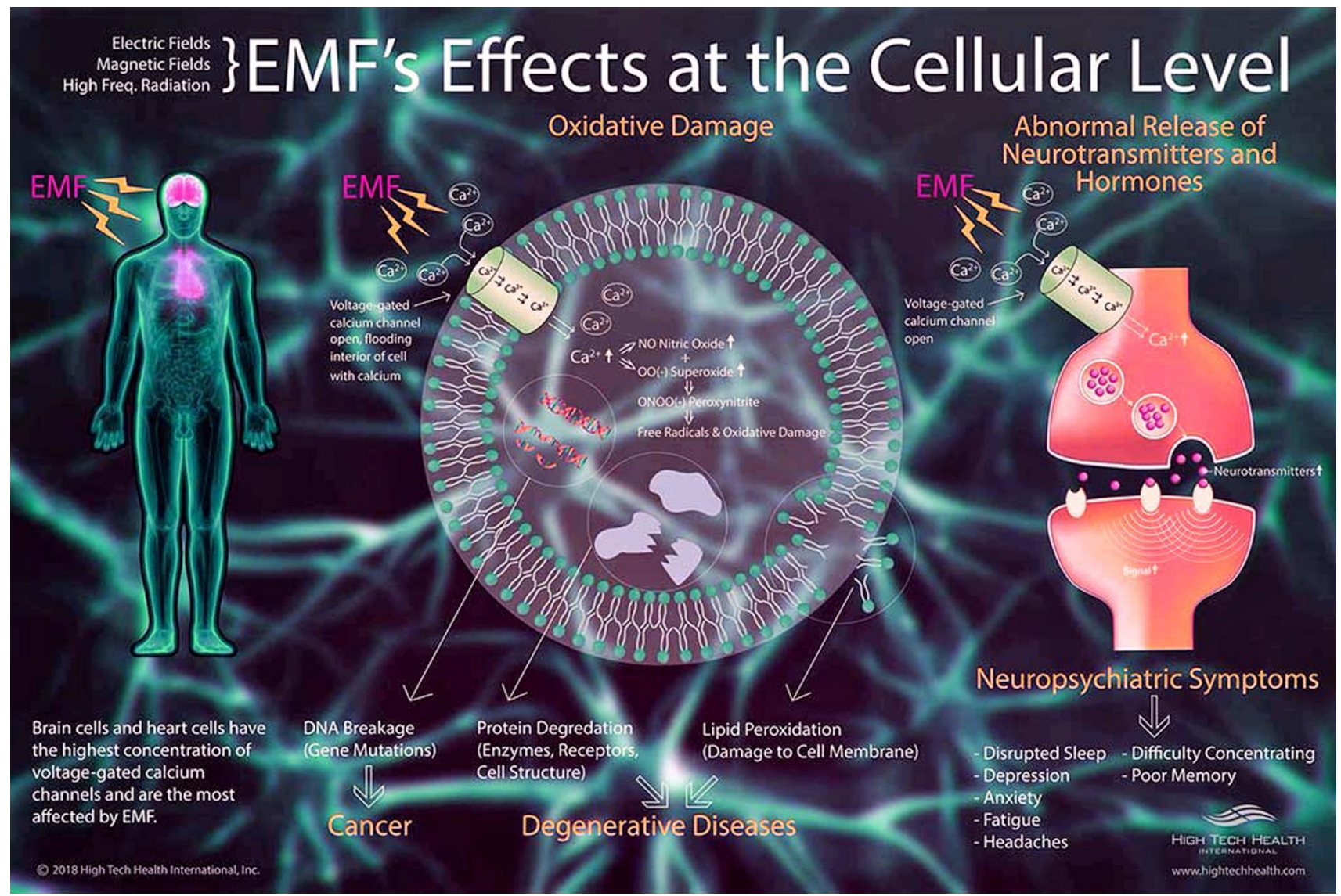

Figure 2: EMF effect at the cellular level 11 . 


\subsection{Electromagnetic seed Rudraksha:}

Rudraksha seeds are also known as Rudraksh and these are the dried seeds of a tree that only grows in a few places. Rudraksha (Elaeocarpus ganitrus) plant is a 50-200-foot-tall huge gigantic plant12, 13. Depending on the area and the type of weather, this tree can reach a height of 14.60 meters to 29.20 meters. Rudraksha seeds are rough and woody on the outside, with a light chocolate color within the berry's pulp. Seeds can be dirty white, yellowish, reddish-brown, or brownish-black in color. Each Rudraksha seed has the altering amount of vertical lines running down from its outside that form 'Mukhs' or face of the seed ${ }^{\mathbf{1 4}}$. The Rudraksha seeds are named appreciated on a base of these faces or 'much. The Rudraksha bead serves as a defensive shield, shielding the wearer from harmful energies.

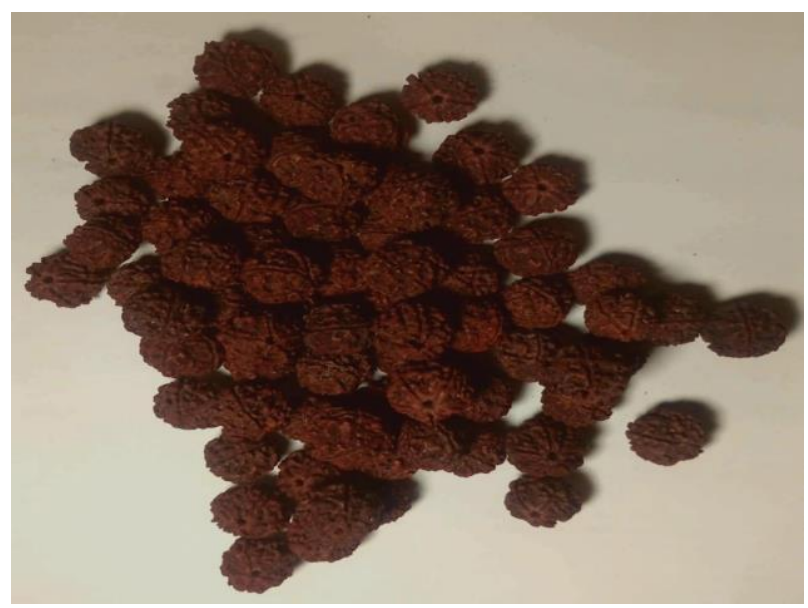

Figure 3: Rudraksha seed ${ }^{18}$.

Wearing Rudraksha seeds heal sicknesses like anxiety, insomnia, nerviness, lack of concentration, despair cardiovascular problems like hypertension, rheumatism, sterility, immune modulator characteristics, also it is noticed that it has also properties like asthma, and anti-aging effect 15, 16. The polarity of Rudraksha beads is Electromagnetic, Paramagnetic, Diamagnetic, and Dynamic. Because of their electromagnetism, Rudraksha also has antiaging properties. Rudraksha Beads interact and integrate with the human body at a subtle level of consciousness while worn. Rudraksha Beads induce and effect positive change in the Bio Electrical System of the Human Body in a lifesupporting manner due to their Dielectric and Magnetic Properties ${ }^{17}$. Positive changes in our Bio-Chemical System occur as a result of this alteration in our body's electrical composition.

\section{MATERIAL AND METHOD:}

This experiment was conducted on ten male human subjects age 20-40 years. This experiment's main objective was to know the effect of unexplained negative electromagnetic fields' harmful effect on the human body and its nullifying effect by Electromagnetic seed (Rudraksha). There are various places on earth where EMF meters detect abnormal electromagnetic fields without any valid cause and sources. In this particular locality where abnormal EMF exist, most of the time people experience a symptom like stress, anxiety, and hypoxia-like condition. We researched to know that whether Rudraksha seeds have any curing effect in such a situation or not. Most of the time the stress and hypoxia condition originates to mental conation and detail of this condition can be studied from the scientific topic of subject parapsychology. We conducted this experiment on a standard Oximeter which is used for Covid-19 patient detection and cure. We calculated the oxygen level and pulse rate of ten human subjects in this unexplained electromagnetic field after keeping the subjects in a particular locality for 1hour of time. We then externally applied 108 seeds Rudraksha seed mala in from of necklace to human subjects and again check the human subjects' pulse rate and oxygen level after 1 hour of time. We check out the reading before treatment and after treatment in human subjects to know if there is any improvement in pulse rate and oxygen level in human subjects. The Oximeter used for the research was Dr. Trust Pulse Oximeter- 217. The EMF meter used for the experiment was from Kkmoon model JVW0869076962769CG - EMF Meter Radiation Monitor (Digital). Collection of authenticating Elaeocarpus ganitrus (Rudraksha) was done by purchasing online from Rudra Center (https://www.rudraksharatna.com/) and we further confirmed its originality by CSIR-NISCAIR'sResearch \& Academics in Science \& Technology Communication New Delhi. Authentication No.NISCAIR/RHMD/Consult/2019/3436-37.

\section{RESULTS:}

Table 1: EMF field and Rudraksha effect on Oxygen level, pulse rate on Human subjects

\begin{tabular}{ccccc}
\hline EMF Reading in $\boldsymbol{\mu}$ T & \multicolumn{2}{c}{ Oximeter reading for (Sp02) } & \multicolumn{2}{c}{ Oximeter reading for (bpm) } \\
\cline { 2 - 5 } & Before treatment & After treatment & Before treatment & After treatment \\
$\mathbf{1 . 9 8}$ & 89 & 94 & 97 & 90 \\
$\mathbf{2 . 0 2}$ & 91 & 92 & 76 & 72 \\
$\mathbf{1 . 6 7}$ & 89 & 89 & 98 & 89 \\
$\mathbf{3 . 4 5}$ & 90 & 91 & 67 & 75 \\
$\mathbf{2 . 5 6}$ & 98 & 97 & 86 & 74 \\
$\mathbf{3 . 1 2}$ & 96 & 97 & 69 & 74 \\
$\mathbf{0 . 8 9}$ & 91 & 89 & 83 & 79 \\
$\mathbf{1 . 3 4}$ & 90 & 93 & 94 & 85 \\
$\mathbf{4 . 1 0}$ & 91 & 90 & 76 & 82 \\
\hline
\end{tabular}

EMF $=$ Electromagnetic field, $\mu \mathrm{T}=$ Unit of EMF microtesla, $\mathrm{SpO} 2=$ Oxygen saturation, $\mathrm{bpm}=$ beats per minute 


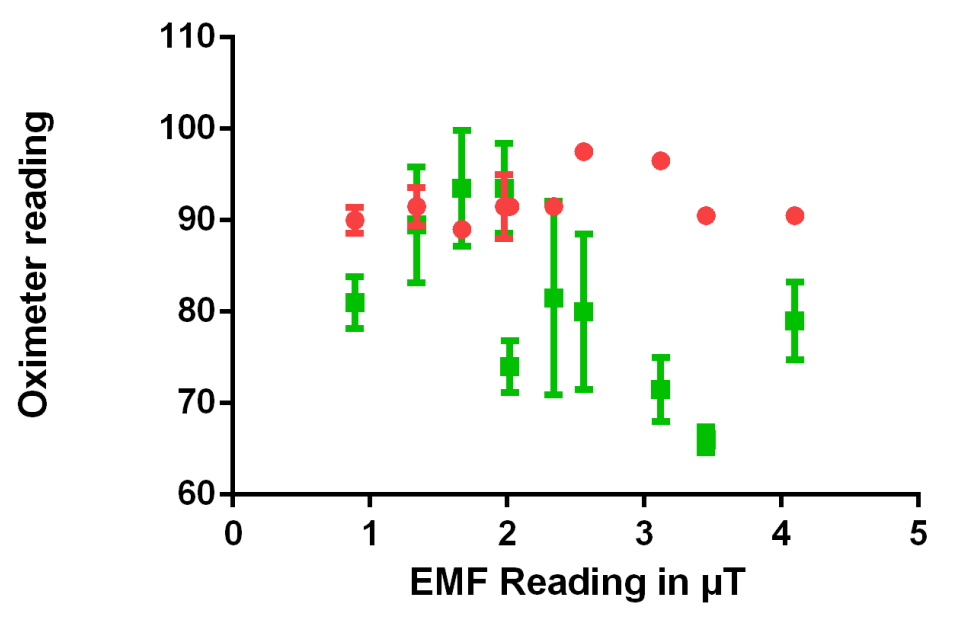

- Oximeter reading for (SpO2)

- Oximeter reading for (bpm)

Figure.4 Graphical presentation Unexplained EMF effect on oxygen level and pulse rate

\section{DISCUSSION:}

The results of this bio-electromagnetic research of unexplained harmful Electromagnetic Radiation influence on Human and its shielding effect by Electromagnetic seed Rudraksha Summarize in the Table.1 of result session in this manuscript. The Result of the research has the close agreement of the Hindu mythology and various traditional pieces of literature that suggested that Rudraksha has protecting effect from the evil spirit and its medicinal effect like Antiageing immunomodulatory effect etc. we expose the human subjects in various places where unexplained Electromagnetic field exist without any valid source. When we exposé our ten human subjects in such condition for one hour they obtain anxiety and shortness of breath, hypoxialike condition. The EMF reading of our Kkmoon digital EMF meter Expressed in microtesla is mention in the first Coolum In table.1. Before treatment with Rudraksha seed, human subjects show low oxygen saturation levels. SpO2 reading of before treatment column show low oxygen saturation level. After treatment with Rudraksha seeds for one hour significant improvement of oxygen saturation level SpO2 obtained which is mentioned in the column of SpO2 level after treatment. Expect few human subjects reading shows that significant improvement in oxygen saturation level and average oxygen saturation level was above 95. When we are much stressed or in anxiety our heart rate and pulse rate increase significantly. Human subjects in this EMF field experience stress anxiety which can be noticed in bpm reading in Oximeter in before treatment condition. The high bpm rate decreases noticeably after treatment with Rudraksha seeds. Table.1 summarizes and discusses the main findings of the research which shows that Rudraksha seed has defiantly some dielectric property that can be used to shield negative EMF field. It improves the SpO2 level and decreased the bpm rate in our body. The results are given in Table.1 and shown graphically in Figure.4. Figre.4 is an XY graph in which X-Axis shows the EMF measurement and YAxis shows the Oximeter reading. The red spot presents the Oximeter reading for $\mathrm{SpO} 2$ and Green Square represents the oximeter reading for $\mathrm{bpm}$. The above figures illustrate the fact Mean and Error and standard deviation of the various parameters. After rigorous examination, it was discovered that Rudraksha seeds have some protecting effect against the EMF field due to their electromagnetic property. From these results, it is clear that Rudraksha seeds' medicinal property is not a myth but its medicinal healing effect is based on its electromagnetic property. By carefully examining the data, it is found that either Rudraksha seed improves our SpO2 or bpm level or it has some effect on Human psychology which helps us feel confident in a negative electromagnetic field. These observations are limited and cannot be extrapolated to the medicinal field until further scientific investigation.

\section{CONCLUSION:}

Elaeocarpus ganitrus seeds Rudraksha traditionally used as a praying seed in Hindu and Buddhism for their positive effect on our mental health. It is believed that Rudraksha seeds drive out negative energy, evil spirit; protect us from harmful planetary effects and boost our self-confidence due to their Paramagnetic and Diamagnetic property. In this scientific research the first time this approach is proposed for use of Rudraksha seeds shielding effect on the EMF field in this scientific experiment. The main conclusions of this work are drawn together and presented in the discussion section. In summary, this novel study shows that Rudraksha seeds may improve our oxygen level and decrease our pulse rate significantly. Rudraksha seeds can be used as alternate medicinal therapy like acupressure, acupuncture, and magnetic therapy. This study design seems to be advantageous compared with the majority of studies Undergone on Rudraksha as these methods directly show their effect on EMF. Further study is needed to uncover the detail of the phenomenon of diamagnetic property of Rudraksha.

\section{CONSENT FOR PUBLICATION:}

Not applicable.

\section{CONFLICT OF INTEREST:}

The authors affirm no conflict of interest, financial or otherwise

AUTHOR CONTRIBUTION: The main author is Mr. Subhashish Tripathy Prepared the article under the supervision of Dr. Arun Kumar Mishra and Dr. Amit Mishra.

\section{ACKNOWLEDGMENTS:}

I would like to express my honest appreciation and deep thanks to Dr. Vikram Kumar Sahoo Director M.P.C.P Kanpur for his motivation for preparing the manuscript. I am also grateful to Dr. Ajay Kumar Products Research \& Indian System of Medicine Lab. Molecular Bio prospection Division 
CSIR for his steadfast support for the preparation of this manuscript.

\section{REFERENCES:}

1. Heald MA, Marion JB. Classical electromagnetic radiation: Courier Corporation; 2012.

2. Smith GS. An introduction to classical electromagnetic radiation: Cambridge University Press; 1997.

3. Read FH. Electromagnetic radiation. Chichester. 1980.

4. Rohrlich F. The definition of electromagnetic radiation. Il Nuovo Cimento (1955-1965). 1961; 21(5):811-22. https://doi.org/10.1007/BF02785607.

5. Kong JA. Theory of electromagnetic waves. New York. 1975.

6. Zamanian A, Hardiman C. Electromagnetic radiation and human health: A review of sources and effects. High Frequency Electronics. 2005; 4(3):16-26.

7. Angell TS, Kirsch A. Optimization methods in electromagnetic radiation: Springer Science \& Business Media; 2004. https://doi.org/10.1007/b97629.

8. Reshetnyak S, Shcheglov V, Blagodatskikh V, Gariaev P, Maslov MY. Mechanisms of interaction of electromagnetic radiation with a biosystem. LASER PHYSICS-LAWRENCE-. 1996; 6:621-53.

9. Pšenáková Z. Numerical modeling of electromagnetic field effects on the human body. 2006.

10. Repacholi MH, editor An overview of WHO's EMF project and the health effects of EMF exposure. Proceedings of the
International Conference on Non-Ionizing Radiation at UNITEN (ICNIR 2003) Electromagnetic Fields and Our Health; 2003.

11. Iralu V, Upadhaya K. Seed dormancy, germination and seedling characteristics of Elaeocarpus prunifolius Wall. ex Müll. Berol.: a threatened tree species of north-eastern India. New Zealand Journal of Forestry Science. 2018; 48(1):16 https://doi.org/10.1186/s40490-018-0121-y.

12. Jain S, Jatwa K, Jain V, Sharma A, Mahajan S. A review on elaeocarpus sphaericus (Rudraksha). Pharma News. 2021.

13. Joshi S, Jain P. A review on ethnomedicinal and traditional uses of Elaeocarpus ganitrus roxb.(rudraksha). Int J Pharm Bio Sci. 2014; 5(1):495-511.

14. Tripathy .S , Mishra. A , Mishra .AK. Rudraksha Medicinal Importance in Ayurveda and Spiritual Healing. International Journal of Pharmaceutical Research. 2020; 12(3):113-31. https://doi.org/10.31838/ijpr/2020.12.03.017.

15. Dennis T. Rudraksha-not just a spiritual symbol but also a medicinal remedy. Sachitra Ayurved. 1993; 46(2):142.

16. Hardainiyan S, Nandy BC, Kumar K. Elaeocarpus ganitrus (Rudraksha): A reservoir plant with their pharmacological effects. Int J Pharm Sci Rev Res. 2015; 34:55-64.

17. Lee DW. The biology of rudraksha. Current Science (Bangalore). 1998;75(1).

18. Tripathy S, Mishra A, Mishra AK. Cytotoxicity, antioxidant and antimicrobial assay of Elaeocarpus ganitrus (Rudraksha) seeds. International Journal of Botany Studies. 2021; 6(2):387-91. 